\title{
Detailed structure of the X-ray jet in 4C 19.44 (PKS1354+195)
}

\author{
D. A. Schwartz, ${ }^{1}$ D. E. Harris,${ }^{1}$ H. Landt,${ }^{1}$ A. Siemiginowska, ${ }^{1}$ \\ E. S. Perlman, ${ }^{2}$ C. C. Cheung, ${ }^{3}$ J. M. Gelbord,${ }^{4}$ D. M. Worrall, ${ }^{5}$ \\ M. Birkinshaw, ${ }^{5}$ S. G. Jorstad,${ }^{6}$ A. P. Marscher ${ }^{6}$ and L. Stawarz ${ }^{7}$ \\ ${ }^{1}$ Smithsonian Astrophysical Observatory, Cambridge MA 02138, USA \\ ${ }^{2}$ University of Maryland, Baltimore County, Baltimore MD 21250, USA \\ ${ }^{3}$ NRAO/Stanford University, Palo Alto CA 94305, USA \\ ${ }^{4}$ Massachusetts Institute of Technology, Cambridge MA 02139, USA \\ ${ }^{5}$ Physics Department, University of Bristol, Bristol BS8 1TL UK \\ ${ }^{6}$ Institute for Astrophysical Research, Boston University, Boston MA 02215, USA \\ ${ }^{7}$ Kavli Inst. for Particle Astroph. and Cosmology, Stanford Univ., Stanford CA 94305, USA \\ email: das@cfa.harvard.edu
}

\begin{abstract}
We investigate the variations of the magnetic field, Doppler factor, and relativistic particle density along the jet of a quasar at $z=0.72$. We chose $4 \mathrm{C} 19.44$ for this study because of its length and straight morphology. The 18 arcsec length of the jet provides many independent resolution elements in the Chandra X-ray image. The straightness suggests that geometry factors, although uncertain, are almost constant along the jet. We assume the X-ray emission is from inverse Compton scattering of the cosmic microwave background. With the aid of assumptions about jet alignment, equipartition between magnetic-field and relativistic-particle energy, and filling factors, we find that the jet is in bulk relativistic motion with a Doppler factor $\approx$ 6 at an angle no more than $10^{\circ}$ to the line of sight over de-projected distances $\approx 150-600 \mathrm{kpc}$ from the quasar, and with a magnetic field $\approx 10 \mu$ Gauss.
\end{abstract}

Keywords. (Galaxies:) quasars: individual (4C 19.44, PKS 1354+135) - galaxies: jets - X-rays

\section{Analysis of the jet}

We present preliminary results from a $200 \mathrm{ks}$ observation of the quasar 4C 19.44 (=PKS 1354+135), using Chandra observation identifications (OBSID's) 6903, 6904, 7302, 7303. We analyze the jet assuming X-ray production by inverse Compton scattering on the cosmic microwave background. This is motivated in the middle panel of Figure 1 since the optical flux for two knots (from Sambruna et al. (2004)) do not allow an extrapolation of the synchrotron radio spectrum to the X-ray region. We follow Tavecchio et al. (2000), and Celotti et al. (2001) to calculate the rest frame magnetic field B and the Doppler factor $\delta=[\Gamma(1-\beta \cos (\theta))]^{-1}$. The formalism requires many assumptions on the physics and on unknown values of parameters. Consistent with our previous work (Schwartz et al. 2006) we assume a minimum total energy in magnetic field and relativistic particles, filling factor $=1$, an electron spectral index $\mathrm{m}=2.4$ with cutoffs at low and high energies tuned to give radio emission from $10^{6} \mathrm{~Hz}$ to $10^{12} \mathrm{~Hz}$, equal energy in electrons and protons, and that the bulk Lorentz factor $\Gamma=\delta$ so that the angle to the line of sight takes on the maximum value, $\approx 1 / \delta$, for a given $\delta$. We take the volume of regions 4 through 14 to be 

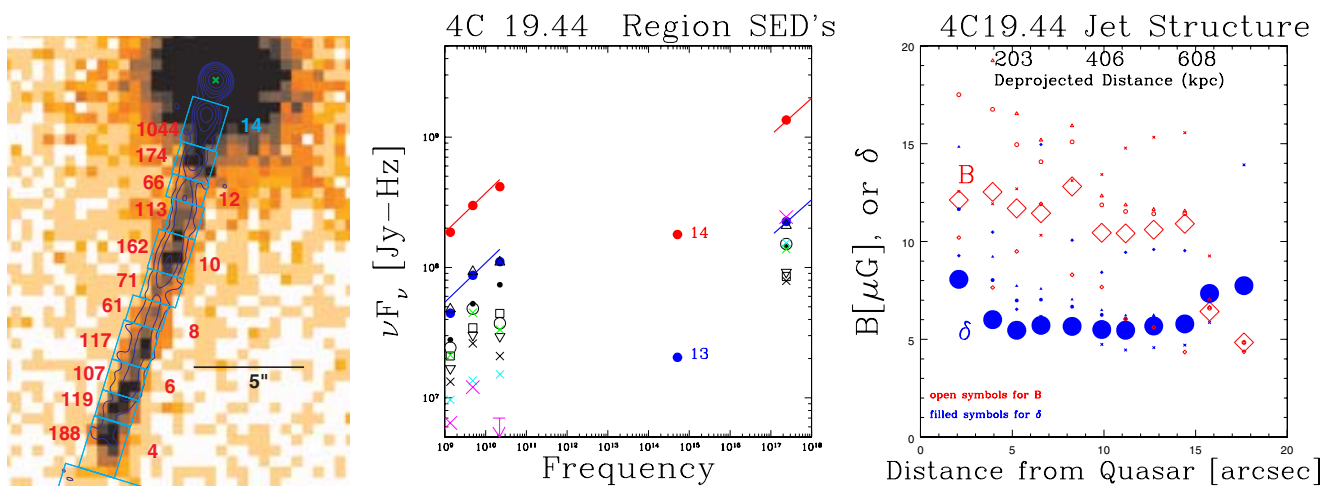

Figure 1. Left panel shows the Chandra 0.5 to $7 \mathrm{keV}$ band X-ray image of the 18 arcsec long jet. The boxes numbered 4 through 14 are an arbitrary division of the jet into distinct regions for independent analysis. Numbers to the left of each box show how many X-ray photons were detected in that region. The contours are of the $4.8 \mathrm{GHz}$ emission. The middle panel shows the spectral energy distributions for these 11 regions. Optical emission is detected only from the regions 14 and 13 (knots A and B of Sambruna et al. 2004). The summed X-ray spectrum of all the regions is consistent with $f_{\nu} \propto \nu^{-0.7}$, and the corresponding $\nu f_{\nu}$ is shown as the solid lines. The optical detection from region 13 can serve as an upper limit to any region, and along with the radio indices prohibits a simple synchrotron spectrum being extrapolated from the radio to $\mathrm{X}$-ray region. The right hand panel shows the results for the magnetic field (large diamonds) and Doppler factor (filled circles) as a function of distance along the jet. Alternate assumptions can lead to systematic changes in these parameters as follows: small crosses use the best-fit power law radio spectral index; small triangles and circles assume conical jets starting at the quasar and $1 \mathrm{kpc}$ from the quasar, respectively, reaching 1" diameter at the end of the jet in each case; small diamonds use the 1.4 to $4.86 \mathrm{GHz}$ spectral index instead of the assumed slope of 0.7.

cylinders of the (resolved) lengths shown in the left panel of Figure 1, and (unresolved) radii assumed to be $0.5=3.62 \mathrm{kpc}$ at the redshift $z=0.72$ of this quasar.

\section{Structure of the Jet}

The results for $\mathrm{B}$ and $\delta$ are presented in the rightmost panel of the figure. With the above assumptions the jet shows similar structure along its length. We give the deprojected distance on the top axis by assuming $\delta=5.5$. We note that if the radii were all $1 \mathrm{kpc}$, the values for B and $\delta$ would be a factor of 1.44 higher. The systematic effects of our assumptions allow alternate profiles, but the numerical values are constrained within a factor roughly 2 . In particular, the increase in $\delta$ at the end of the jet would be peculiar in the IC/CMB scenario. For the baseline values of $\mathrm{B}$ and $\delta$, we can derive the kinetic power, the minimum electron Lorentz factor, and the relativistic electron density as a function of distance along the jet. For de-projected distances 200 to $600 \mathrm{kpc}$ from the quasar, regions 13 to 6 , we require a cutoff to the electron spectrum at energies below $80 m c^{2}$ and we estimate a kinetic power of approximately $(1-2) \times 10^{46} \mathrm{erg} \mathrm{s}^{-1}$, and a total relativistic electron density of roughly $(2-3) \times 10^{-8} \mathrm{~cm}^{-3}$.

We acknowledge support of NASA contract NAS8 39073 to SAO, CXC grants GO56116B, GO6-7111A, GO6-7111B, and HST grant HST-GO-10762.01-A.

\section{References}

Celotti, A., Ghisellini, G. \& Chiaberge, M. 2001, MNRAS, 321, L1

Sambruna, R. M. et al. 2004, ApJ, 608, 698

Schwartz, D. A. et al. 2006, ApJ, 640, 592

Tavecchio, F., Maraschi, L., Sambruna, R. M. \& Urry, C. M. 2000, ApJ, 544, L23 